\title{
Cutting and pasting constrained boundary features
}

\author{
M. Ranta ${ }^{1}, M$. Mäntyläl, M. Inui ${ }^{2}$, and F. Kimura ${ }^{3}$ \\ 'Laboratory of Information Processing Science \\ Helsinki University of Technology \\ Tekniikantie 12, Innopoli, 02150 Espoo 15, Finland \\ tel +358-0-4354 3967, fax +358-0-43543965 \\ emailmra@cs.hut.fi
}

${ }^{2}$ Department of System Engineering, Ibaraki University

Naka-narusawa-cho 4-12-1, Hitachi-shi, Ibaraki 316, Japan

tel +81-249-35-6101 ext 409, fax +81-294-32-1546;

e-mail inui@sumire.dse.ibaraki.ac.jp

${ }^{3}$ Department of Precision Machinery Engineering, University of Tokyo

Hongo 7-3-1, Bunkyo-ku, Tokyo 113, Japan

tel $+81-3-3812-2111$ ext 6455, fax $+81-3-3812-8849$,

e-mail kimura@cim.pe.u-tokyo.ac.jp

\begin{abstract}
This paper presents a feature modelling approach that allows the designer to reuse former design solutions in the definition of new design features. Instead of the conventional approach of providing the designer a pre-defined set of basic volumetric features to build parts with, the proposed system offers utilities for cutting portions of former models as new boundary features that can be pasted into new product models. The boundary features are based on an ordinary boundary representation model that has been extended, or relaxed, in order to allow the manipulation of open boundary portions, i.e., incomplete solids that lack some faces and thus have no volume. The size and position dimensions of the boundary features are presented and managed with a constraint system that is attached to control the geometry information of the boundary representation model. A small prototype system has been implemented to demonstrate the suggested approach.
\end{abstract}

\section{Keywords}

Design features, boundary features, reuse of former solutions, presentation of incomplete geometry, dimensioning, constraints 


\section{INTRODUCTION}

The difficulty of feature modelling lies in defining a feature concept that falls between generally applicable geometric modelling and application dependent feature hierarchies. Predefined feature libraries restrict the designers too much and there is an obvious need for interactive feature definition tools. This paper goes even further and understands design features to be a method for supporting reuse of former design solutions. In addition to a common library of standard features, each designer may collect his/her own feature catalogue by selecting, or cutting, portions of existing product models to be pasted into new products.

Boundary representation models have been proven to be a suitable basis for feature modelling since they provide easy access to faces and their attributes. However, the potential of boundary manipulation is so far not properly exploited as the emphasis has been on volumetric operations related to manufacturing features. Various non-manifold models, for example (Rossignac 90) and (Gursoz 91), have been presented to extend modelling capabilities from conventional solid models towards tools for managing mixed-dimensional models. The proposed cut and paste approach requires utilities for manipulating a particular type of non-manifolds that correspond to open boundary portions or sets of faces that are the basis for boundary features.

An essential characteristic of feature based systems is parametric modelling. According to a common approach a feature is pre-defined with a certain set of size parameters that are passed as arguments to a procedure that generates the volumetric model. The utilisation of constraints allows more flexible dimension driven geometry such as suggested in (Gossard 88), which proposes relative position operators for dimensions so that changes in dimension values can be automatically translated as changes in geometry and topology. Other examples of constraint based approaches are (Kimura 86) and (Suzuki 88) which propose a declarative method for generating geometry and features according to a constraint representation of a higher level. In this paper constraints are proposed as a methodology for managing the sizing and positioning of boundary features.

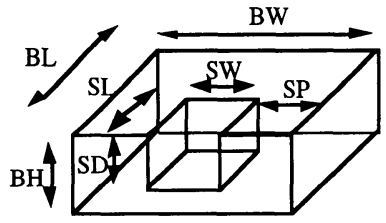

a) the original part

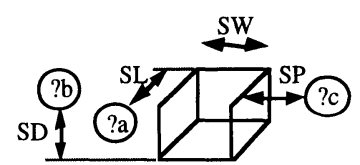

b) cut feture

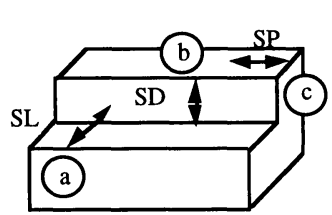

c) positioning to another part

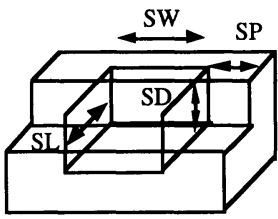

d) "floating feature"

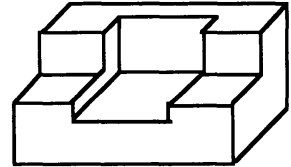

e) pasted feature

Figure 1 An example of cutting and pasting a constrained boundary feature.

The rest of this paper is organized as follows. First, we give an overview of the design process supported by constrained boundary features. Section 3 gives an outline of the architecture of the implemented prototype system. Next, the constraint model used in this work and its connection with the boundary representation model is described. Section 5 describes the cut and paste operations for constrained boundary features. Finally, we give our conclusions and discuss topics for further research. 


\section{DESIGN WITH CONSTRAINED BOUNDARY FEATURES}

A new boundary feature is defined by selecting a set of faces and cutting the boundary portion as shown in the example of defining a slot-like feature in Figures 1a and $b$. If the definition is based on a plain boundary model the designer must first explicitly add the dimension constraints to the model. The resulting constrained boundary feature is an incomplete solid with broken constraints as shown in Figure 1b. The open edges and broken constraints maintain default information on the neighbour and reference faces, which allows an unattached feature be modified by changing its size dimensions such as the width of the slot.

Figure $1 \mathrm{c}$ and $1 \mathrm{~d}$ demonstrate the pasting of a feature. First, the designer repairs the positioning constraints by replacing the default reference faces with proper ones according to the new environment and assigns proper values to the positioning dimensions. Figure 1c shows how the slot length is set to refer to the front face of the part and slot depth to the top face and finally the position is related to the right hand side face of the part. Next, the designer accepts the positioning and the system adjusts the boundary and constraint models together. As Figure 1d shows the border edges of the feature are replaced by new ones calculated according to the intersection of the surfaces of the meeting faces.

Figure 2 shows how a feature structure is useful in maintaining a constrained boundary model. When the designer removes the slot of Figure 2a the hole becomes "floating" since it was originally positioned with constraints referring to the faces of the removed slot. The designer may now either delete the hole or repair the positioning by attaching the constraints to the remaining faces of the part as Figure $2 \mathrm{c}$ shows.

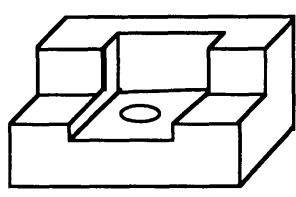

a) a slot is removed

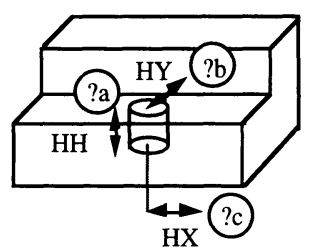

b) a"floating" hole

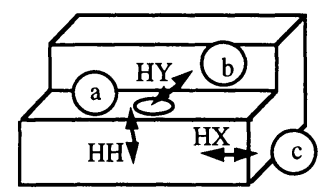

c) repaired positioning constraints

Figure 2 Repairing constraints of the hole after removing the slot.

The explained cut and paste based design process is a simplified one and only intends to give a clear overview of the design style. In real applications the constrained boundary features are typically much more complicated and larger entities which shows the powerfulness of the reuse approach as new products are created by attaching together just a few large design and function oriented features.

\section{CUT AND PASTE BASED FEATURE MODELLING SYSTEM}

The architecture of the prototype system consists of a form feature modeller, a dimension feature modeller, and a feature catalogue as shown in Figure 3. The presented applications correspond to the current implementation; however, a realistic feature modelling environment requires tools for also other aspects such as assembly modelling and manufacturing.

The form feature modeller offers access to feature-based and geometric modelling operations. The geometric modelling operations have been built upon the ACIS modeller. This boundary representation modeller has been extended to allow manipulation of boundary portions that do not necessarily have to be solids. The boundary manipulation operations are in turn the basis for cut and paste tools for boundary representation of shapes.

The dimension feature modeller facilitates manipulation of dimension values and definition of constraints for maintaining dimensions. The DeltaBlue algorithm (Freeman- 
Benson 88) has been used as a foundation for developing a dimensioning system that controls constraints relating geometric entities, i.e., mainly distances of surface variables. The constraint model controls the dimensions of the boundary representation model and thus the models are in frequent interaction.

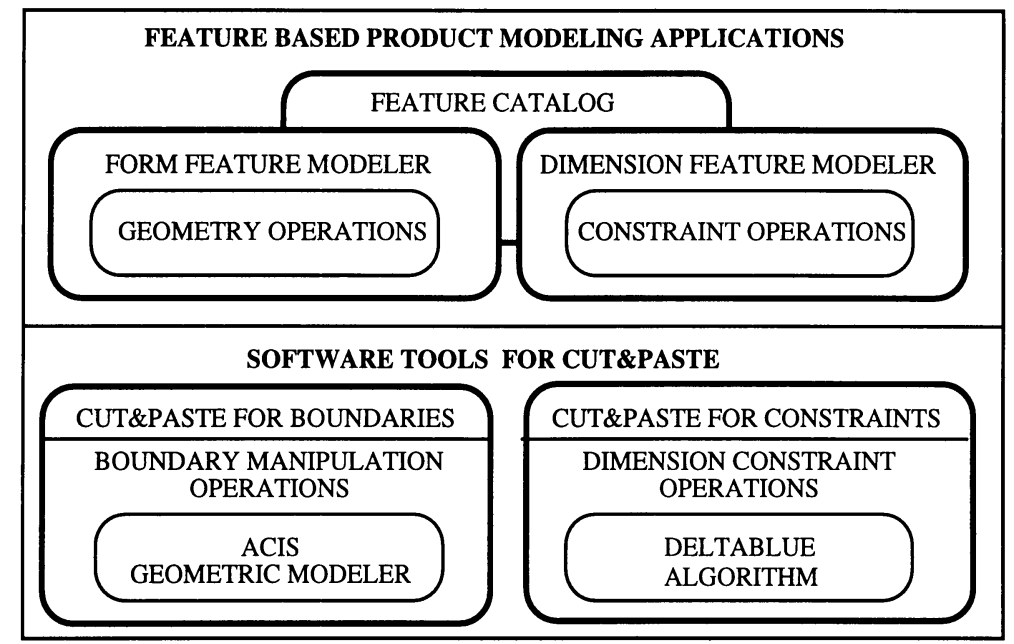

Figure 3 A feature modelling environment.

\section{ATTACHING CONSTRAINS TO A BOUNDARY MODEL}

The cut and paste approach was first implemented for boundary features as explained in (Ranta 93). The designer may select portions of former boundary representation models and reuse them in new designs. Boundary features are usually not volumetric entities but rather correspond to a sets of faces that form an incomplete or open solid. They can be pasted in a quite flexible manner together by adjusting the open faces and edges according to the intersections of surfaces as shown in Figure 1. However, the scaling possibilities of boundary features are poor and, moreover, their locations are determined by clumsy reference coordinate systems. Both of the shortcomings emphasise the need for proper facilities for managing engineering dimensions.

In order to make boundary features parametric and positioning easier a constraint based dimension feature modeller is suggested. The constraint modeller is founded on the DeltaBlue Algorithm (Freeman-Benson 88,90 ) that is a fast incremental constraint solver for satisfying dynamically changing constraint hierarchies. Its successor SkyBlue has been improved to support multi-output methods and allow cycles of constraints to be constructed, although it may not be able to solve the cycles (Sannella 92).

Constraints allow the designer to state declaratively a relation that is to be maintained. Figure 4 shows the operations for expressing the slot length constraint (SL) of Figure 1a. First, two variables are created to store references to the two faces that are to be constrained, i.e., the end face of the slot and the front face of the blank. A third variable is created for representing the value of the constrained length dimension. The Controllervariable and LongVariable classes demonstrate how SkyBlue allows the variables to contain arbitrary data, not just numbers. Both of them are subclasses of variable class that records the information needed by the constraint solver, for example a list of all constraints that reference to the variable and the constraint that presently defines the variable. 
Next, a constraint is created as an instance of the DistanceConstraint class to bind the distance between the two faces to the length value. Each constraint is labelled with a strength indicating how important it is to satisfy it; in the example the distance constraint is given the strongest $S_{-}$required priority. The constraint hierarchy guides the constraint satisfaction algorithm to leave weaker constraints unsatisfied if needed for keeping stronger ones satisfied when the model is overconstrained. In the case of an underconstrained hierarchy, the user can add weak constraints to suggest which solution is chosen. Similarly to the variable class, also the parent class Constraint of DistanceConstraint records other information needed by the constraint satisfaction algorithm, such as a list of variables that are constrained by the constraint. Furthermore, each constraint class provides access to the possible methods for enforcing the constraint. For instance, the methods of DistanceConstraint provide procedures for calculating the LongVariable value according to the distance of the ControllerVariable surfaces, or for moving the Controllervariable surfaces to a distance determined by the LongVariable value. The appropriate method is chosen incrementally by the constraint satisfaction algorithm.

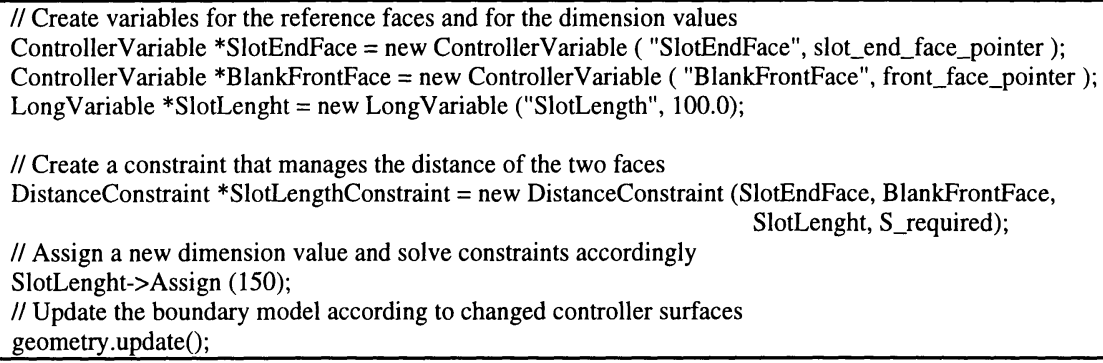

Figure 4 Creating a constraint on slot length and modifying the length value.

In this way the dimensioning constraint operations allow the construction of a model that controls the relative locations of the surfaces of some faces. The system is able to maintain a solution to the constraints incrementally when constraints are added or deleted, or when new values are assigned to the dimensions. As the last row of Figure 4 suggests, it is necessary to integrate the constraint model to the geometric model to make the dimensioning affect it.

Figure 5 shows how the connection between a boundary representation model and a constraint model is built through a surface interface. A Controllervariable of the constraint model contains a pointer to a surface record that belongs to the data structure of the geometric model which thus changes according to the dimensioning constraints.

(Kimura 86) proposes a natural way to present dimensions of 3-D objects by setting constraints on surfaces. The approach uses solid models for shape description and logical formulae for representing geometric constraints on the solid model elements. We propose a similar approach that is extended to support dimensioning of incomplete solids and to utilize feature-based presentation that makes distinction between sizing and positioning constraints.

The left hand side of Figure 5 illustrates the data structure of a boundary representation model (Mäntylä 88) which represents the topology of a part in terms of shells, faces, loops, coedges, edges, and vertices. Geometric information is represented by attaching surface information to faces, curve information to edges, and point information to vertices. We assume that the topology of a solid remains unchanged and that dimension modifications affect only the geometry of a part. This assumption is acceptable since topological changes would change the character and functionality of a part or a feature in a crucial way. Thus, changes in dimensioning affect only geometric information, and in the current state of our implementation, particularly surface information. When the constraint modeller has propagated some dimension changes to the surfaces, the rest of the geometry is updated 
accordingly. Curves are updated by recalculating the intersection of the surfaces of the faces that meet at the edges. Accordingly points are updated by recalculating the intersection of the curves of the edges that meet at the vertices. Thus, the constraint model and boundary representation model are integrated into a constrained boundary model.

It is possible to extend the interface to other entities carrying geometric information, such as curves and points. At any rate, the principle is that dimensioning affects the geometric information while the topology is maintained.

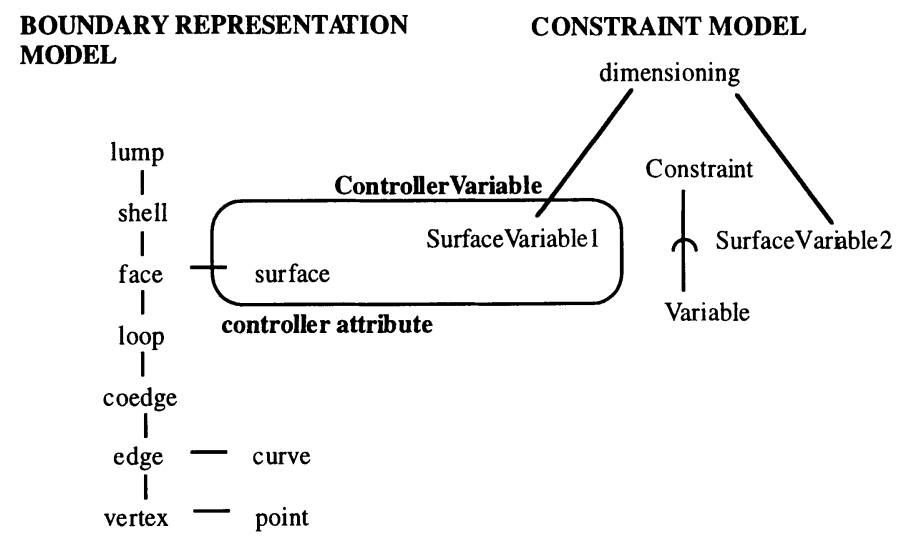

Figure 5 Interface of the boundary representation model and constraint model.

\section{CUT AND PASTE OPERATIONS}

The cut and paste operations are implemented in an analogous way for different types of models according to the pattern shown in Figure 6. In the case of the constraint model the intersection relation corresponds to a constraint and the entity references are surface variables. The cutting $(6 a->b)$ of a constraint occurs when its surface variables are separated into different boundary models as a result of the cutting of a boundary model. During the paste $(6 b->a)$ of a constrained boundary feature the constraints are treated first to determine the positions for the pasting of boundary models.

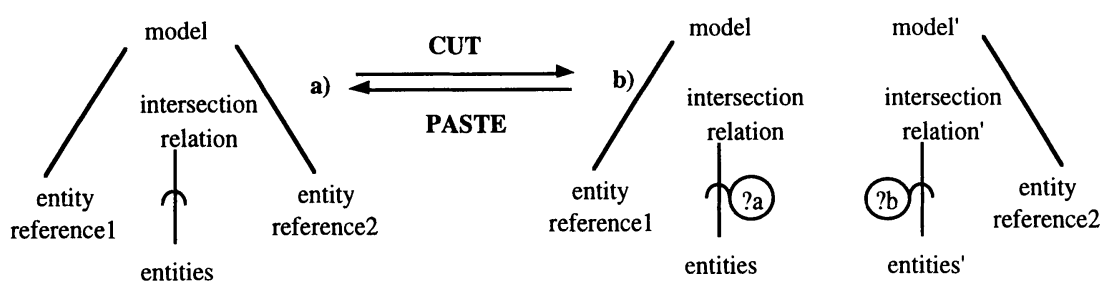

Figure 6 Cutting operation $a->b$ and pasting operation $b->a$.

In the case of the boundary representation model, the intersection relation of Figure 6 corresponds to an edge which relates two halfedges corresponding to the two faces that meet at it. When an edge is cut $(6 a->b)$, the result is two open edges that belong to different models and lack one halfedge as well as a face from one side. The paste operation (6b->a) of two boundary models includes a adjustment that modifies the neighbouring edges to fit each other. 
Figure 7 explains the stages of the cutting algorithm for integrated boundary representation and constraint models. In the case of cutting, the boundary model is the basis for user interaction and thus precedes and controls the cutting of the constraint model. Details of the cutting of a boundary feature are explained in (Ranta 93). After the boundary feature has been separated the constraints are classified according to their surface variables. If both controller surfaces of a constraint belong to the boundary feature the constraint classified local to the feature, i.e., it is a size definition. Accordingly when both of the controller surfaces belong to the product the constraint is local to the part and sets a constraint on its size. Such local constraints are simply attached to the appropriate boundary model.

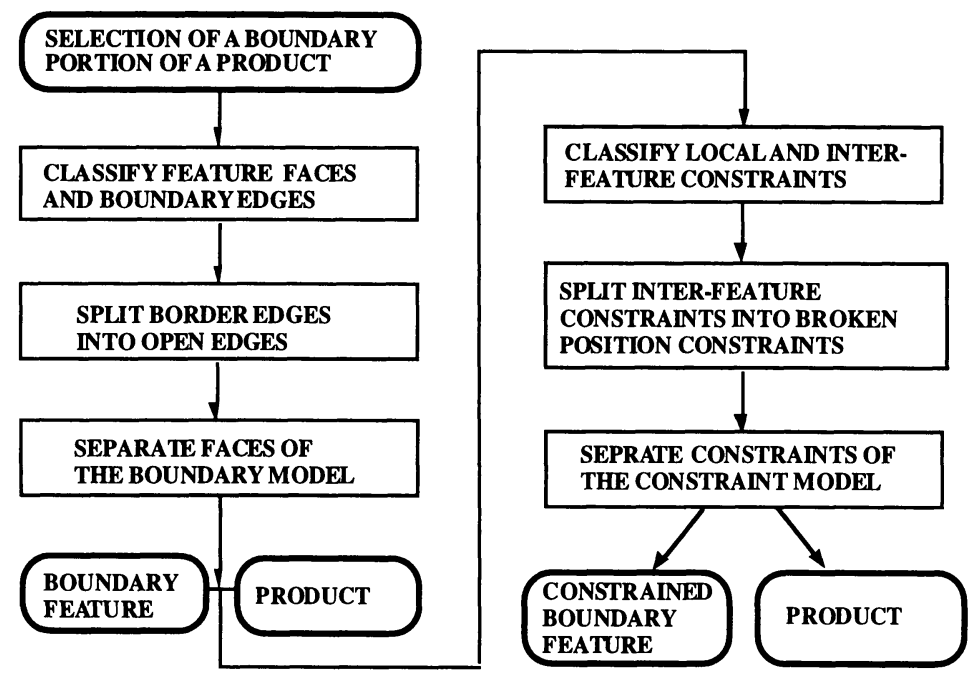

Figure 7 Cutting first the boundary model and then constraint model accordingly.

If one of the surface variables of a constraint belongs to the boundary feature and the other to the product it is an inter-feature constraint, i.e., it is a position definition that defines a relation across feature limits. Such inter-feature constraints are cut according to Figure 6 into two broken constraints that are classified so that one belongs to the feature and the other to the product. The ?a and ?b symbols denote that the cutting left the constraints with default surfaces on the place of one of their controller variables. The $a$ ? and $b$ ? default variables do no longer have pointers to any actual faces, but just carry a copy of the former surface definition.

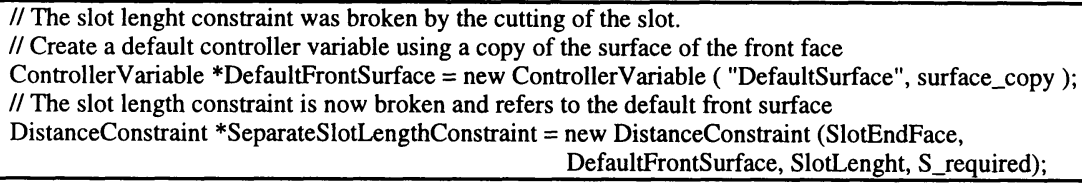

Figure 8 Generating a default surface controller when the constraint model is cut.

Figure 8 shows operations related to the cutting the length constraint that takes place when the slot of Figure 1 is cut from the blank. First, a default controller variable is created to store a copy of the surface of the blank's front face. Then a length constraint of the separated slot is defined to refer to the default surface variable. 
Finally the constraints are separated into groups that are attached to the appropriate boundary model. The result is a constrained boundary feature with open edges and broken positioning constraints that are to be repaired while the feature is pasted.

Figure 9 demonstrates the pasting algorithm for an integrated boundary representation and constraint model. In case of pasting the constraint model is the basis for user interaction and thus precedes and controls the cutting of the boundary representation model. First, the designer may change the size of the constrained boundary feature by assigning values to its size constraints and see the effect on the updated boundary model.

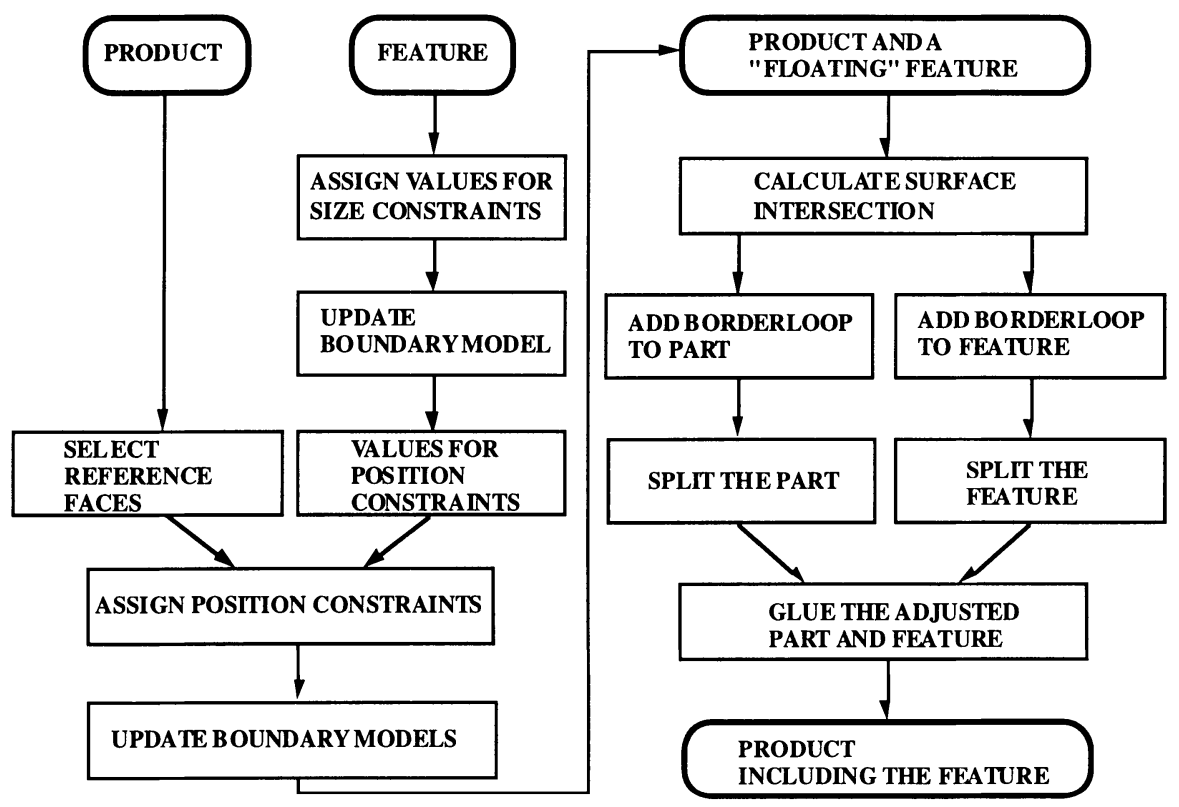

Figure 9 Pasting first position constraints and then boundary models accordingly.

Next, the designer repairs the positioning constraints by selecting new reference faces to the positioning constraints and setting appropriate values to position dimensions. This attaches the constraint models of the feature and the product to into one constraint hierarchy. Figure 10 shows how the default controller variable is assigned with a front face from the new blank and the geometry is updated to be able to display the slot floating at its planned position.

$$
\begin{aligned}
& \text { // Position the slot according to a face from its new environment by assigning it to the controller variable } \\
& \text { DefaultFrontSurface - > Assign(new_front_face_pointer); } \\
& \text { // Update the boundary model according to changed controller surfaces } \\
& \text { geometry.update(); }
\end{aligned}
$$

Figure 10 Assigning a new reference face to position the feature in a new environment.

At this stage, the boundary representation models are still separate. The constrained boundary feature is positioned according to its new environment and "floats" in its future location. The designer may now iterate the editing of feature size and position until he/she is happy and ready to finally let the system to paste the boundary models into one product model wholeness. The right hand side of figure 9 demonstrates how the boundary feature and 
product are adjusted to each other and then glued together. Details of the pasting of boundary features are presented in (Ranta 93).

\section{CONCLUSIONS}

The proposed cut and paste approach provides the designer an interaction method that is more obvious and produces less surprising results than the set operations on volumetric entities. The constraint based sizing and positioning allows the designer to apply the familiar practices of engineering dimensioning in a straightforward manner instead of constructing less practical systems of reference coordinates. Thus, the cut and paste interaction style was found suitable both boundary representation and constraint feature modelling.

The proposed constraint feature model extends former approaches to allow the management of incomplete solids. Furthermore, it experiments the presentation of broken constraints. Although the current prototype offers a limited set of possible constraints it suggests, the advantages of the capability to represent incomplete states during the design process. .Product models that are incomplete as solids and features that float according to constraints allow the designer to postpone design decisions to an appropriate time.

If beforehand standardisation is considered to be the crucial characteristic of features it is questionable whether the proposed approach can be regarded as feature modelling. However, particularly in the case of design features, the advantages of user defined features and an evolving feature library seem to be well justified, since designers find it easier to work with large function related features than basic general ones.

Constrained boundary modelling does not necessarily require any feature concept, but may be applied as an interaction method as such without any recording of the feature structure at all. However, the recording of the feature structure is practical for manipulating relationships of features, maintaining meaning of shapes, and so on.

The cut and paste approach has so far been implemented for a boundary representation model and a constraint model. The analogy in cutting and pasting these models was closer than expected which is a promising result when further extensions of the cut and paste approach are considered. In the future the system should manage other types of product information as well, for example tolerance, material and assembly information. Such variety of viewpoints is essential as an ultimate goal of feature based product modelling.

\section{REFERENCES}

Freeman-Benson, B.N. and Maloney, J. (1988) The DeltaBlue Algorithm: an incremental constraint hierarchy solver. Technical report 88-11-09, Department of Computer Science, University of Washington, Seattle, Washington, 98195, USA.

Freeman-Benson, B.N., Maloney, J. and Borning, A, (1990) An incremental constraint solver. Communications of the ACM, January 1990, 33(1), 54-63.

Gossard, D.C., Zuffante, R.P. and Sakurai, H. (1988) Representing dimensions, tolerances, and features in MCAE systems. IEEE Computer Graphics and Applications, 8(8), 51-59.

Gursoz, E.L., Choi, Y. and Prinz, F.B. (1991) Boolean set operations on non-manifold boundary representation objects. CAD 23(1), 33-39.

Kimura, F., Suzuki, H. and Wingård, L. (1986) A uniform approach to dimensioning and tolerancing in product modelling. In Computer Applications in Production and Engineering, CAPE '86, (ed. K. B $\varnothing$, L. Estensen, P. Falster and E.A. Warman), 165-178. IFIP, Elsevier Publishers (North-Holland).

Mäntylä, M. (1988) An introduction to solid modelling. Computer Science Press, College Park, Maryland. 
Ranta, M., Inui, M., Kimura, F. and Mäntylä, M. (1993) Cut and paste based modelling with boundary features. Proceedings of the Second Symposium on Solid Modelling and Applications (ed. J. Rossignac, J. Turner and G. Allen), ACM, Montreal, Canada.

Rossignac, J. and O'Connor, M. (1990) Selective geometric complex: a dimensionindependent model for pointsets with internal structures and incomplete boundaries. Geometric Modelling for Product Engineering (ed. M. Wozny, J.U. Turner and K. Preiss), North-Holland.

Sannella, M. (1992) The SkyBlue constraint solver. Technical Report 92-07-02, Department of Computer Science, University of Washington, Seattle, Washington, 98195, USA.

Suzuki, H., Ando, H. and Kimura, F. (1988) Synthesizing product shapes with geometric design constraints and reasoning. Proceedings of IFIP WG 5.2 2nd Workshop on Intelligent $\mathrm{CAD}$, North-Holland, Amsterdam.

\section{BIOGRAPHY}

Mervi Ranta is a research scientist at the Laboratory of Information Processing Science in the Helsinki University of Technology. She is currently working on a design feature approach that promotes the reuse of product knowledge. She received her MS in Computer Science from Helsinki University of Technology in 1987. During 1988-1992 she was a visiting researcher at the Kimura Laboratory in the Department of Precision Machinery Engineering of the University of Tokyo. Her research interests include feature based product modeling, geometric modeling, early design, and concurrent engineering.

Martti Mäntylä received his Dr.Eng. in 1983 at the Helsinki University of Technology. In 1983-1984 he was a visiting scholar with the Computer Systems Laboratory at the Stanford University. In 1989 he was a World Trade Visiting Scientist at the IBM Thomas J. Watson Research Center. Currently he is a Professor of Information Technology with the Laboratory of Information Processing Science at the Helsinki University of Technology, Finland, where he is the head of research teams in product modeling and realization, rapid prototyping, assembly robotics, usability engineering, and analysis and measurement of CIM systems. Mäntylä's research interests include the full range of computer applications in engineering, such as CAD, CAM, computer graphics, user interfaces, and data base management. He is a member of the editorial board of Computer-Aided Design (Butterworth), an associate editor of the ACM Transactions on Graphics and a member of the ACM, the IEEE Computer Society, and the Eurographics Association, where he is also a Member of the Executive Committee. He is also a member of IFIP working groups 5.2, 5.3, and 5.10.

Masatomo Inui is an associate professor in the Department of System Engineering of the Ibaraki University. He was a lecturer in the Department of Precision Machinery Engineering of the University of Tokyo from 1991 to 1993. His interests include solid modelling machining and process-planning automation, design and manufacturing process modelling, product modelling, and geometric reasoning. He received his MSEng. degree in information engineering from the University of Tokyo in 1986, and a Dr.Eng. in precision machinery engineering from the University of Tokyo in 1991.

Fumihiko Kimura is a professor in the Department of Precision Machinery Engineering of the University of Tokyo. He was a research associate at the Electrotechnical Laboratory of the Ministry of International Trade and Industry from 1974 to 1979 . He then moved to the University of Tokyo, and was an associate professor from 1979 to 1987 . He has been active in the fields of solid modeling, free-form surface modelling and product modelling. His research interests now include the basic theory of CAD/CAM and CIM, concurrent engineering, engineering simulation and virtual manufacturing. He is involved in the product model data exchange standardization activities of ISO/TC184/SC4, and is a member of IFIP WG 5.2 and 5.3 , and a corresponding member of CIRP. He graduated from the Department of Aeronautics, the University of Tokyo, in 1968, and received a Dr. Eng. Sci. degree in aeronautics from the University of Tokyo in 1974. 